\title{
BIOLOGICAL EVALUATION, QSAR AND MOLECULAR MODELING STUDIES OF 2,4-DICHLOROBENZOIC ACID DERIVATIVES AS ANTIMICROBIAL AGENTS
}

\author{
SAMRIDHI THAKRAL, VIKRAMJEET SINGH* \\ Department of Pharmaceutical Sciences, Guru Jambheshwar University of Science and Technology, Hisar-125001, Haryana, India. \\ Email: vikramjeetsinghjudge@gmail.com
}

Received: 22 January 2019, Revised and Accepted: 18 February 2019

\section{ABSTRACT}

Objective: The aim of this study was to evaluate 2,4-dichlorobenzoic acid derivatives as antimicrobial agents through in vitro, QSAR and molecular docking studies.

Methods: The compounds were subjected to in vitro antimicrobial screening by test tube dilution method and the structural characteristics governing the antimicrobial potential were studied using QSAR methodology. These compounds were also screened for docking simulation to find out binding confirmation of reported compounds with PDB 1aj0 and 5fsa using AutoDock tools and discovery studio.

Results: The antimicrobial evaluation data indicated that compounds 13 and 18 were found to be the most effective against all the bacterial strains and Aspergillus niger while compounds 1 and 14 exhibited more antifungal potential against Candida albicans. QSAR studies confirmed the role of molar refractivity and Balaban index (J) as controlling parameters for antimicrobial potential. Molecular modeling study revealed that compounds interact with the active site of PDB by hydrophobic, hydrogen bonding, and Van der Wall interactions.

Conclusion: These test compounds were identified as potent candidates for the control of microbial strains tested, and structural relationship with activity may provide valuable information for further design and synthesis of compounds with antimicrobial potential.

Keywords: Antimicrobial, QSAR, Molecular docking.

(c) 2019 The Authors. Published by Innovare Academic Sciences Pvt Ltd. This is an open access article under the CC BY license (http://creativecommons. org/licenses/by/4. 0/) DOI: http://dx.doi.org/10.22159/ajpcr.2019.v12i4.31631

\section{INTRODUCTION}

The multidrug resistance associated with life-threatening microbial infections is a re-emerging and alarming microbial threat and amenable for millions of deaths annually due to the inadequacy of efficacious antimicrobial drugs [1]. To improve the current antimicrobial therapies, several studies have been conducted to improve the available treatments, but due to the continuance of the antibiotic resistance, pathogens have become a critical and complicated health issue [2]

Sulfonamides, being antibacterial, having the prominent mechanism of action including attachment of sulfonamides with the dihydropteroate synthetase (DHPS) enzyme and alteration of bacterial pathways of folic acid synthesis in few eukaryotic cells, but in human beings, this mechanism is not followed $[3,4]$. The sulfonamides have been recognized due to various reported biological activities such as anticancer [5-9], anti-Alzheimer [10,11], anti-tubercular [12,13], antimicrobial [14-17], anti-inflammatory [18], carbonic anhydrase inhibitors [19-22], antidiabetic [11], anticonvulsant [23], and antimalarial [24-26].

Due to these facts, there is a prime need to shorten the duration of therapy and ascertain newer antimicrobial agents to avert the emergence of resistance. In continuation of our efforts dedicated toward development of antimicrobials, we have screened compounds (1-18) reported by Thakral and Singh [27] for antimicrobial potential along with QSAR and computational studies.

\section{METHODS}

\section{Antimicrobial evaluation}

The reported compounds were evaluated for their in vitro antimicrobial potential against bacterial and fungal strains through serial dilution technique to get their minimum inhibitory concentration [28]. The weighed amount of nutrient broth and Sabouraud dextrose broth was dissolved in distilled water to prepare nutrient medium for bacteria and fungus, respectively, and $1 \mathrm{ml}$ of nutrient medium was transferred to each test tube. The test sample compound $(0.01 \mathrm{~g})$ was dissolved in $10 \mathrm{ml}$ of dry dimethyl sulfoxide to give a stock solution of $100 \mu \mathrm{g} / \mathrm{ml}$. The $1 \mathrm{ml}$ solution of test compounds was transferred to test tubes having sterilized nutrient medium and diluted serially to get a set of five dilutions of test compounds having concentrations 50, 25, 12.5, 6.25, and $3.125 \mu \mathrm{g} / \mathrm{ml}$. Minimal inhibitory concentration for each sample was investigated against Gram-positive bacterial strains; Bacillus subtilis (MTCC 441), Staphylococcus aureus (MTCC 7443), and Staphylococcus epidermidis (MTCC 435), two Gram-negative bacterial strains; Escherichia coli (MTCC 1652) and Pseudomonas aeruginosa (MTCC 424), and two fungal strains; Aspergillus niger (MTCC 8189) and Candida albicans (MTCC 227). The freshly cultured strains of each organism were transferred in to test tubes and incubated at $37 \pm 1^{\circ} \mathrm{C}$ for $24 \mathrm{~h}$ for bacterial strains, $48 \mathrm{~h}$ for $C$. albicans and 7 days at $25 \pm 1^{\circ} \mathrm{C}$ for A. niger. Ciprofloxacin was considered as a standard for antibacterial and fluconazole for antifungal activity.

\section{QSAR}

The two-dimensional structures were drawn, and energy minimization was done by molecular mechanics force field (MM) process of hyperchem 6.03 (1993), and the pre-optimization of the energy minimized structures was done. The calculation of physicochemical parameters for each compound was done using TSAR 3.3 software for Windows (2000) and the SPSS software package (1999) was used for regression analysis $[29,30]$

\section{Molecular modeling}

The advanced docking program, AutoDock Vina was used to evaluate the binding properties of test compounds into microbial targets [31]. The crystal structures of a ternary complex of $E$. coli dihydropteroate 
synthase (1aj0) [32] and sterol 14-alpha-demethylase (CYP51) from a pathogenic yeast Candida albicans in complex with the antifungal drug posaconazole (5fsa) $[33,34]$ were downloaded from the protein data bank (www.rcsb.org). All bound water molecules and cocrystallized ligands were removed from protein, and polar hydrogen atoms were added. 2D structure of ligands was penciled in MarvinSketch and saved in pdb format, then further saved into pdbqt format using AutoDock Vina. Energy minimization was performed using the MMFF94 force field. Then docking was performed according to specified conditions of grid box used by AutoDock tools. The Vina search space chosen was center_x $=39.66$, center_y $=7.14$, center_z $=2.63$; center_x $=193.6$, center $\_y=1.0$, center_ $z=39.0$ and size $\_x=40$, size $y=40$, size $\_z=40$ for 1aj0, 5fsa, respectively. The exhaustiveness was set to be 8 .

\section{RESULTS AND DISCUSSION}

\section{In vitro antimicrobial studies}

All the compounds were evaluated for their in vitro antibacterial activity against $S$. aureus, B. subtilis, and S. epidermidis (Gram-positive bacteria), and E. coli and P. aeruginosa (Gram-negative bacteria), and antifungal potential against $C$. albicans and A. niger. The power management integrated circuit (pMIC) values obtained are recorded in Table 1.

Based on results, it could be inferred that compounds 13 and 18 have the highest and broad spectrum of antimicrobial potential while compound 2 exerted the highest activity against $S$. epidermidis with a pMIC value of $1.785 \mu \mathrm{M}$. Compounds 1, 3, and 11 exhibited good antimicrobial activity against all strains. Compounds 1 and 14 exhibited the most potent antifungal activity against $C$. albicans with a pMIC value of $2.102 \mu \mathrm{M}$. All other test compounds showed moderate antimicrobial potential against all bacterial and fungal strains. Compounds 13 and 18 also displayed more inhibition effect against $A$. niger species. Compounds 1, 3, 11, and 14 exhibited good antifungal potential against $A$. niger.

It is observed that in this series compound 2 with $\mathrm{p}$ - $\mathrm{Cl}$ substituent showed selective inhibition against $S$. epidermidis whereas the activity was diminished when substituted with the $\mathrm{o}-\mathrm{NO}_{2}$ group. Results revealed that chloro substituent on phenyl ring exerted higher activity against all microbial strains as compared to fluoro substituent. Apparently, the compound bearing methoxy group displayed more antimicrobial potential as compared to methyl substituted compound. Replacement of aromatic anilines with aliphatic amines as observed in compounds 15,16 , and 17, afforded a decrease in antimicrobial potential against all strains. A deep insight into the structure of test compounds revealed that their antimicrobial activity seems to be modulated by the effect of the substituent. Above all, it could be inferred that a significant broad spectrum of antimicrobial activity was allied with compounds incorporating electron withdrawing substitution [17].

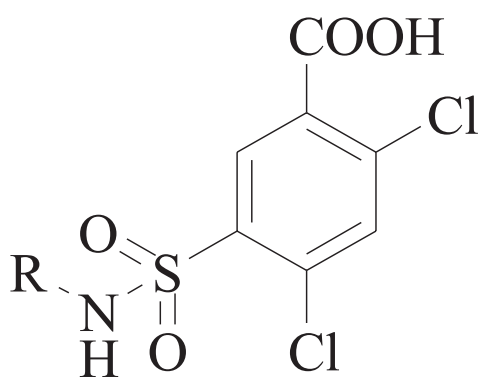

General structure of 2,4-dichlorobenzoic acid derivatives.

\section{QSAR study}

In this present study, dataset of 18 compounds (1-18) was used to establish a quantitative relationship between antimicrobial activity and structural descriptors of substituted sulfonamide derivatives. The structural descriptors coding for lipophilic, steric, electronic, and molecular connectivity were used in this study, and the corresponding value for each molecular descriptor is presented in Table 2. A correlation matrix was constructed to find out the inter-relationship of different calculated molecular descriptors and antimicrobial activity as well as interrelationship of different molecular descriptors with each other (Table 3).

It was observed from correlation matrix that highest correlation existed between Randic parameter (R) and first-order molecular connectivity index $\left({ }^{1} \chi, r=1.000\right)$ and between Weiner index $(\mathrm{W})$ and zero-order molecular connectivity index $\left({ }^{0} \chi, r=0.995\right)$. The least interrelationship was observed for the energy of highest occupied molecular orbital (HOMO) and energy of lowest unoccupied molecular orbital $(\mathrm{r}=0.050)$ and energy of HOMO and third-order alpha shape indices $\left(\mathrm{k} \alpha_{3,} \mathrm{r}=-0.063\right)$. In general high inter-relationship was observed for different calculated molecular descriptors with each other. The correlation of molecular descriptors with respective antibacterial and antifungal activity is presented in Table 4 .

The different molecular descriptors were subjected to linear free energy regression analysis with antibacterial activity against $S$. aureus and a mathematical model (Eq. 1) illustrating the importance of molar refractivity (MR) was obtained.

$\mathrm{pMIC}_{\mathrm{sa}}=0.052 \mathrm{MR}+1.039$

$\mathrm{n}=18, \mathrm{r}=0.970, \mathrm{r}^{2}=0.940, \mathrm{q}^{2}=0.929, \mathrm{~s}=0.009, \mathrm{~F}=256.482$

The QSAR model represented by Equation 1 is monoparametric and based on the number of compounds included in the dataset and the rule of thumb directed us to go for development of multiparametric models which can quantify the activity of test molecules against $S$. aureus. The dataset was subjected to multiple linear regression analysis and Equation 2 was obtained which depicted the role of the energy of HOMO along with MR in modulating the observed antibacterial against $S$. aureus.

$\mathrm{pMIC}_{\mathrm{saMLR}}=0.0055 \mathrm{MR}-0.0068 \mathrm{HOMO}+0.954$

$\mathrm{n}=18, \mathrm{r}=0.974, \mathrm{r}^{2}=0.949, \mathrm{q}^{2}=0.926, \mathrm{~s}=0.0087, \mathrm{~F}=139.817$

An effort was made to further enhance the $r$ value of QSAR model represented by Equation 2 and a detailed investigation of this model depicted that compound 6 was acting as an outlier whose response values were outside the expected limits.Hence, Compound 6 was excluded from the study (being an outlier), and a new QSAR model was obtained, represented by Equation 3, with improved $r, r^{2}, q^{2}, F$ values, and low $S$ values.

$\mathrm{pMIC}_{\mathrm{saMLR}}=0.0054 \mathrm{MR}-0.0044 \mathrm{HOMO}+0.983$

$\mathrm{n}=17, \mathrm{r}=0.981, \mathrm{r}^{2}=0.963, \mathrm{q}^{2}=0.937, \mathrm{~s}=0.0077, \mathrm{~F}=181.325$

Equation 3 was used to predict the antibacterial activity of test compounds against $S$. aureus and a comparison of observed, predicted and residual antibacterial activity is presented in Table 5.

The results presented in Table 5 revealed that low residual values for antibacterial activity were obtained using QSAR model represented by Equation 3 which suggest that the developed model was a valid one. Same QSAR models were obtained for antibacterial activity of test compounds against B. subtilis, P. aeruginosa, E. coli, and antifungal activity against $A$. niger.

To develop QSAR model for antifungal activity of test compounds against $C$. albicans, the entire dataset was subjected to regression analysis and Equation 4 was obtained in which Balaban index (J) was found to be effectively controlling the antifungal activity.

$\mathrm{pMIC}_{\mathrm{ca}}=-0.414 \mathrm{~J}+2.729$

$n=18, r=0.643, r^{2}=0.414, q^{2}=0.325, s=0.131, F=11.300$

The $r$ value in Equation 4 was 0.643 which was less than the required limit $(r \geq 0.7)$ and the outcomes of Equation 4 were studied in detail to 
Table 1: In vitro antimicrobial activity of 2,4-dichlorosubstituted derivatives in terms of pMIC values ( $\mu \mathrm{mol} / \mathrm{ml}$ )

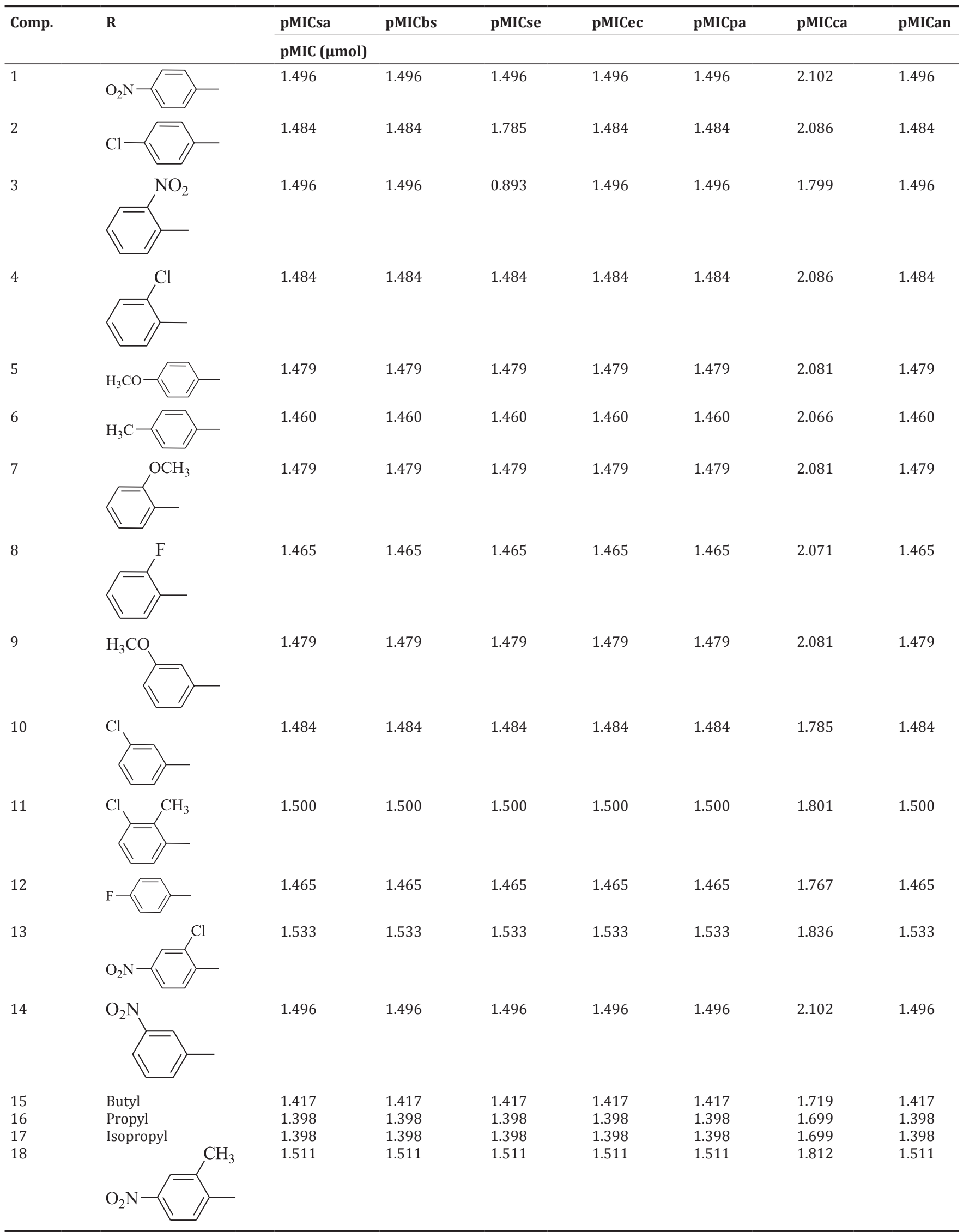

pMIC: Power management integrated circuit 


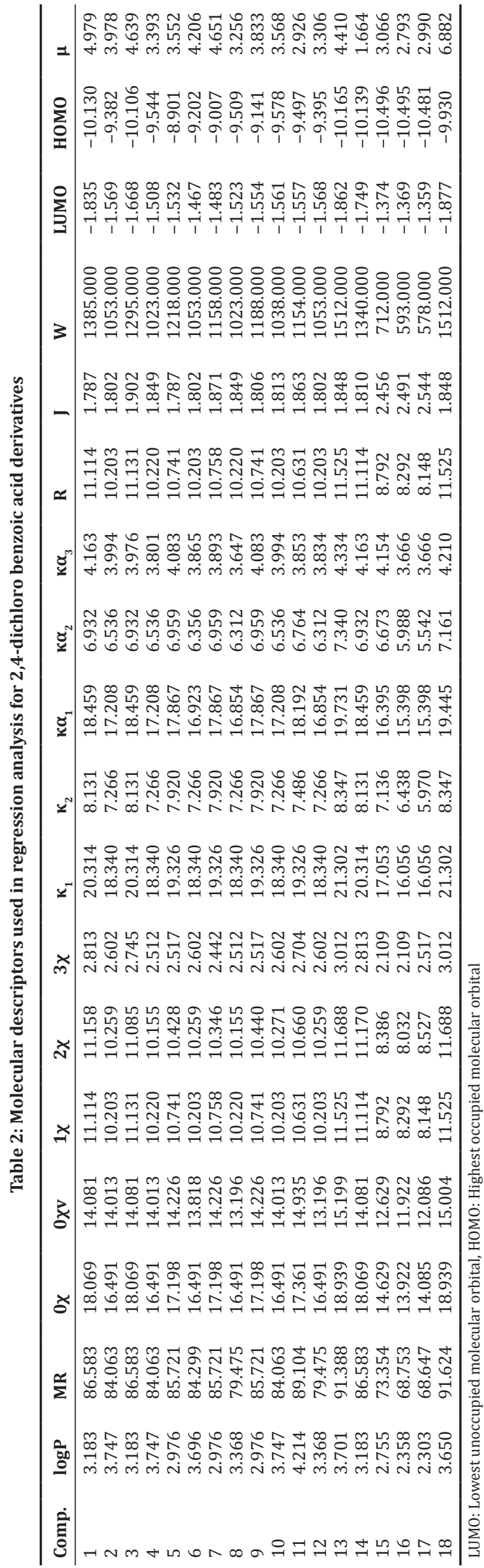

find out the reason for low $r$ value and it was found that compounds 3 , $10,11,12$, and 18 were behaving as outliers as their response values were outside the experimental limits of dataset and QSAR model represented by Equation 5 was built up excluding these five compounds.

$\mathrm{pMIC}_{\mathrm{ca}}=0.525 \mathrm{~J}+3.015$

$n=13, r=0.922, r^{2}=0.851, q^{2}=0.817, s=0.068, F=62.730$

The QSAR model represented by Equation 5 has been built up using 13 compounds and it has got high $\mathrm{r}, \mathrm{r}^{2}, \mathrm{q}^{2}, \mathrm{~F}$ values, and low $\mathrm{S}$ values, which supported the fact that model represented by Equation 5 was valid and it can be used for prediction of antifungal activity of target compounds against $C$. albicans, and the predicted antimicrobial activity is presented in Table 5. Valid QSAR models were not obtained for antibacterial activity of test compounds against $S$. epidermidis.

\section{Docking study}

In silico molecular docking studies were executed to investigate the possible potential binding modes for these sulfonamide derivatives against microbial targets. In the present study, H-bonding, hydrophobic interactions, and free binding energy say, and dock score were considered for the analysis (Table 6).

In the case of antibacterial activity, all the docked compounds showed binding energy from $-6.8 \mathrm{kcal} / \mathrm{mol}$ to $-8.6 \mathrm{kcal} / \mathrm{mol}$. In the binding mode of topmost active, compound 13 potently bound to 1aj0 through four hydrogen bonding and hydrophobic interactions (Fig. 1). Hydrogen bonding interactions are as: The oxygen atom of $\mathrm{SO}_{2} \mathrm{NH}$ formed one hydrogen bond with Ser:222 (2.56 $)$, hydrogen atom of $\mathrm{COOH}$ created hydrogen bond with Thr:177 (2.50 ̊) whereas oxygen atom of carbonyl of $\mathrm{COOH}$ engaged with Gly:191 (2.57 $\AA$ ) amino acid residue and oxygen atom of $\mathrm{NO}_{2}$ interacted with Arg:220 amino acid residue with $3.20 \AA$ bond length. In case of hydrophobic interactions, $\mathrm{Cl}$ of 2,4-dichlorobenzoic acid was involved in alkyl interactions with Ala:151, Pro: 152 whereas $\mathrm{Cl}$ of 2-Cl,4- $\mathrm{NO}_{2}$ substituted phenyl ring interacted with Lys:221 amino acid residues. Pi-alkyl interactions were formed by 2,4-dichloro substituted phenyl ring and 2-Cl, 4- $\mathrm{NO}_{2}$ substituted ring with Ala:151 and Pro:64, Lys:221 amino acid residues, respectively. Ser:222 amino acid was also found to engage with 2-Cl, 4- $\mathrm{NO}_{2}$ substituted phenyl ring through pi-sigma interaction.

Second most active compound 18 showed six hydrogen bonding and hydrophobic interactions. Compound 18 maintained four hydrogen bonding interactions same as compound 13 but two additional bonds formed by hydrogen atom of $\mathrm{COOH}$ with Asn: 144 with a bond length of $1.84 \AA$ and protonated oxygen of $\mathrm{NO}_{2}$ with $\mathrm{Arg}: 220$ with a bond length of $2.29 \AA$. In a comparison of compounds $8(\mathrm{o}-\mathrm{F})$ and 12 (p-F), halogen bond was established by ortho substituted fluorine may be contributing to the better activity of compound 8. Comparison of 2 (p-Cl) and 12 (p-F), 2 formed five hydrogen bond interactions whereas, in compound 12 , three hydrogen bonds were observed. Compound 2 also formed two additional hydrophobic interactions such as alkyl interaction with Lys:221 (4.80 $)$ ) and pi-alkyl interaction with Phe:190 (4.28 $\AA$ ) by chlorine which resulted in increased in the activity of compound 2. In a comparison of compounds $5\left(\mathrm{p}-\mathrm{OCH}_{3}\right), 7\left(\mathrm{o}-\mathrm{OCH}_{3}\right)$, and $9\left(\mathrm{~m}-\mathrm{OCH}_{3}\right)$, pi-pi T-shaped interaction was only observed in compound 7 with Phe: 190 amino acid residue (4.87 ̊̊).

In the case of antifungal activity, all the compounds displayed binding energy from $-6.6 \mathrm{kcal} / \mathrm{mol}$ to $-8.4 \mathrm{kcal} / \mathrm{mol}$. The foremost active compound 1 formed two hydrogen bonding, hydrophobic and Van der Wall interactions with active binding site (Fig. 2). The protonated nitrogen of $\mathrm{NO}_{2}$ created hydrogen bond with His:468 (2.12 $\AA$ ) amino acid residue while $\mathrm{NH}$ of $\mathrm{SO}_{2} \mathrm{NH}$ engaged with Pro:462 (2.24 $\left.\AA\right)$ amino acid residue. $\mathrm{NO}_{2}$ substituted phenyl ring established amidepi-stacked interaction with Phe:463, pi-alkyl interaction with Ile:379 and pi-sulfur interaction with Cys: 470 amino acid residues. The $\mathrm{Cl}$ of 2,4-dichloro substituted phenyl ring formed alkyl interaction with 
Table 3: Correlation matrix for pMICsa with molecular descriptors of 2,4-dichloro benzoic acid derivatives

\begin{tabular}{|c|c|c|c|c|c|c|c|c|c|c|c|c|}
\hline & $\log P$ & MR & $\mathbf{0} \chi$ & $1 \chi$ & $2 \chi$ & $\kappa \alpha_{3}$ & $\mathbf{R}$ & J & $\mathbf{W}$ & LUMO & HOMO & pMICsa \\
\hline $\log P$ & 1.000 & & & & & & & & & & & \\
\hline MR & 0.718 & 1.000 & & & & & & & & & & \\
\hline $0 \chi$ & 0.565 & 0.948 & 1.000 & & & & & & & & & \\
\hline $1 \chi$ & 0.587 & 0.959 & 0.989 & 1.000 & & & & & & & & \\
\hline $2 \chi$ & 0.634 & 0.942 & 0.985 & 0.977 & 1.000 & & & & & & & \\
\hline$\kappa \alpha_{3}$ & 0.180 & 0.587 & 0.623 & 0.589 & 0.552 & 1.000 & & & & & & \\
\hline $\mathrm{R}^{3}$ & 0.587 & 0.959 & 0.989 & 1.000 & 0.977 & 0.589 & 1.000 & & & & & \\
\hline W & 0.529 & 0.936 & 0.995 & 0.985 & 0.976 & 0.667 & 0.985 & -0.828 & 1.000 & & & \\
\hline LUMO & -0.422 & -0.748 & -0.881 & -0.829 & -0.876 & -0.682 & -0.829 & 0.629 & -0.897 & 1.000 & & \\
\hline HOMO & 0.435 & 0.493 & 0.329 & 0.440 & 0.367 & -0.063 & 0.440 & -0.702 & 0.325 & 0.050 & 1.000 & \\
\hline pMICsa & 0.728 & 0.970 & 0.961 & 0.959 & 0.965 & 0.583 & 0.959 & -0.863 & 0.948 & -0.838 & 0.382 & 1.000 \\
\hline
\end{tabular}

LUMO: Lowest unoccupied molecular orbital, HOMO: Highest occupied molecular orbital

Table 4: Correlation of molecular descriptors with antimicrobial activity of 2,4-dichloro benzoic acid derivatives

\begin{tabular}{|c|c|c|c|c|c|c|c|}
\hline & pMICsa & pMICbs & pMICse & pMICec & pMICpa & pMICca & pMICan \\
\hline $\log \mathrm{P}$ & 0.728 & 0.728 & 0.298 & 0.728 & 0.728 & 0.261 & 0.728 \\
\hline MR & 0.970 & 0.970 & 0.098 & 0.970 & 0.970 & 0.534 & 0.970 \\
\hline $0 \chi$ & 0.961 & 0.961 & -0.031 & 0.961 & 0.961 & 0.492 & 0.961 \\
\hline $0 \chi v$ & 0.952 & 0.952 & 0.157 & 0.952 & 0.952 & 0.459 & 0.952 \\
\hline $1 \chi$ & 0.959 & 0.959 & -0.001 & 0.959 & 0.959 & 0.561 & 0.959 \\
\hline $1 \chi \mathrm{v}$ & 0.951 & 0.951 & 0.166 & 0.951 & 0.951 & 0.464 & 0.951 \\
\hline $2 \chi$ & 0.965 & 0.965 & 0.015 & 0.965 & 0.965 & 0.505 & 0.965 \\
\hline $3 \chi$ & 0.823 & 0.823 & 0.021 & 0.823 & 0.823 & 0.269 & 0.823 \\
\hline $3 \chi v$ & 0.561 & 0.561 & 0.262 & 0.561 & 0.561 & 0.075 & 0.561 \\
\hline$\kappa_{1}$ & 0.934 & 0.934 & -0.073 & 0.934 & 0.934 & 0.452 & 0.934 \\
\hline$\kappa_{2}^{1}$ & 0.861 & 0.861 & -0.085 & 0.861 & 0.861 & 0.504 & 0.861 \\
\hline$\kappa_{3}^{2}$ & 0.757 & 0.757 & -0.023 & 0.757 & 0.757 & 0.427 & 0.757 \\
\hline$\kappa \alpha_{1}$ & 0.938 & 0.938 & -0.026 & 0.938 & 0.938 & 0.386 & 0.938 \\
\hline$\kappa \alpha_{2}$ & 0.816 & 0.816 & -0.006 & 0.816 & 0.816 & 0.404 & 0.816 \\
\hline$\kappa \alpha_{3}^{2}$ & 0.583 & 0.583 & 0.122 & 0.583 & 0.583 & 0.201 & 0.583 \\
\hline$J$ & -0.863 & -0.863 & -0.211 & -0.863 & -0.863 & -0.671 & -0.863 \\
\hline W & 0.948 & 0.948 & 0.007 & 0.948 & 0.948 & 0.515 & 0.948 \\
\hline $\mathrm{Te}$ & -0.948 & -0.948 & 0.036 & -0.948 & -0.948 & -0.417 & -0.948 \\
\hline ElE & -0.953 & -0.953 & 0.097 & -0.953 & -0.953 & -0.438 & -0.953 \\
\hline NuE & 0.951 & 0.951 & -0.106 & 0.951 & 0.951 & 0.440 & 0.951 \\
\hline LUMO & -0.838 & -0.838 & -0.024 & -0.838 & -0.838 & -0.314 & -0.838 \\
\hline HOMO & 0.382 & 0.382 & 0.316 & 0.382 & 0.382 & 0.596 & 0.382 \\
\hline$\mu$ & 0.381 & 0.381 & -0.148 & 0.381 & 0.381 & 0.231 & 0.381 \\
\hline
\end{tabular}

Table 5: Comparison of observed, predicted, and residual activity of 2,4-dichloro benzoic acid derivatives

\begin{tabular}{|c|c|c|c|c|c|c|}
\hline \multirow[t]{2}{*}{ Comp } & \multicolumn{3}{|c|}{ pMICsaMLR (Eq. 3) } & \multicolumn{3}{|c|}{ pMICca (Eq. 5) } \\
\hline & obs & pre & res & obs & pre & res \\
\hline 1 & 1.496 & 1.495 & 0.001 & 2.102 & 2.077 & 0.025 \\
\hline 2 & 1.484 & 1.478 & 0.006 & 2.086 & 2.069 & 0.017 \\
\hline 3 & 1.496 & 1.495 & 0.001 & - & - & - \\
\hline 4 & 1.484 & 1.479 & 0.005 & 2.086 & 2.045 & 0.041 \\
\hline 5 & 1.479 & 1.485 & -0.006 & 2.081 & 2.077 & 0.004 \\
\hline 6 & - & - & - & 2.066 & 2.069 & -0.003 \\
\hline 7 & 1.479 & 1.486 & -0.007 & 2.081 & 2.034 & 0.047 \\
\hline 8 & 1.465 & 1.454 & 0.011 & 2.071 & 2.045 & 0.026 \\
\hline 9 & 1.479 & 1.486 & -0.007 & 2.081 & 2.068 & 0.013 \\
\hline 10 & 1.484 & 1.479 & 0.005 & - & - & - \\
\hline 11 & 1.500 & 1.506 & -0.006 & - & - & - \\
\hline 12 & 1.465 & 1.454 & 0.011 & - & - & - \\
\hline 13 & 1.533 & 1.522 & 0.011 & 1.836 & 2.046 & -0.210 \\
\hline 14 & 1.496 & 1.495 & 0.001 & 2.102 & 2.065 & 0.037 \\
\hline 15 & 1.417 & 1.425 & -0.008 & 1.719 & 1.726 & -0.007 \\
\hline 16 & 1.398 & 1.401 & -0.003 & 1.699 & 1.708 & -0.009 \\
\hline 17 & 1.398 & 1.400 & -0.002 & 1.699 & 1.680 & 0.019 \\
\hline 18 & 1.511 & 1.522 & -0.011 & - & - & - \\
\hline
\end{tabular}

Table 6: The Docking score of reported compounds with PDB (1aj0, 5fsa)

\begin{tabular}{lll}
\hline Comp & $\mathbf{1 a j 0}$ (Kcal/mol) & $\mathbf{5 f s a}$ (Kcal/mol) \\
\hline 1 & -8.5 & -8.4 \\
2 & -8.0 & -8.1 \\
3 & -8.4 & -7.7 \\
4 & -8.1 & -7.9 \\
5 & 7.5 & -7.9 \\
6 & -8.2 & -8.3 \\
7 & -8.3 & -7.9 \\
8 & -8.6 & -8.0 \\
9 & -7.9 & -7.8 \\
10 & -7.9 & -7.9 \\
11 & -8.3 & -8.0 \\
12 & -8.4 & -8.1 \\
13 & -8.5 & -8.4 \\
14 & -8.2 & -8.1 \\
15 & -6.8 & -6.8 \\
16 & -6.8 & -6.6 \\
17 & -6.9 & -6.7 \\
18 & -8.6 & -8.1 \\
Ciprofloxacin & -8.2 & - \\
Fluconazole & - & -6.9 \\
\hline
\end{tabular}




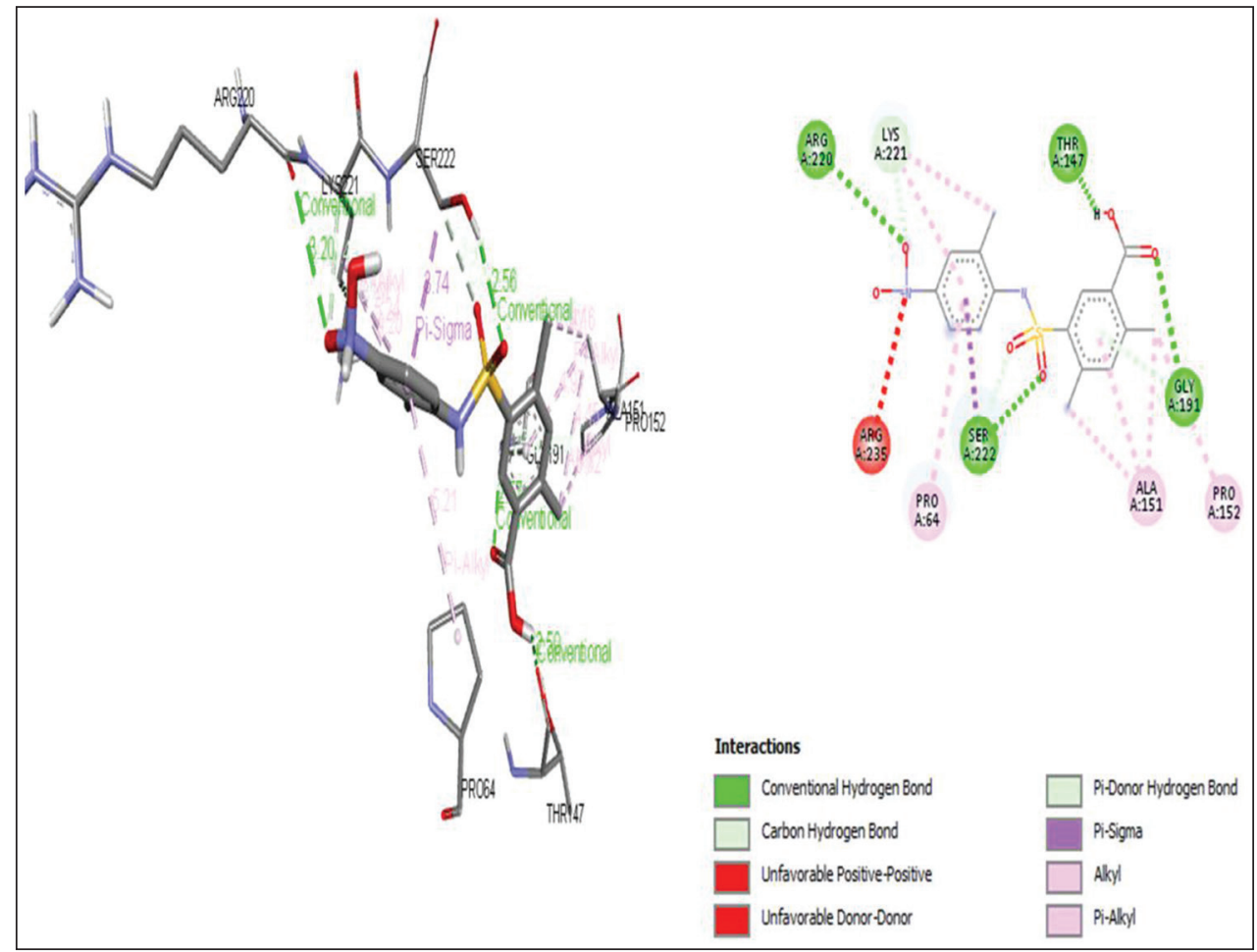

Fig. 1: Binding interactions of compound 13 with the active site of 1aj0

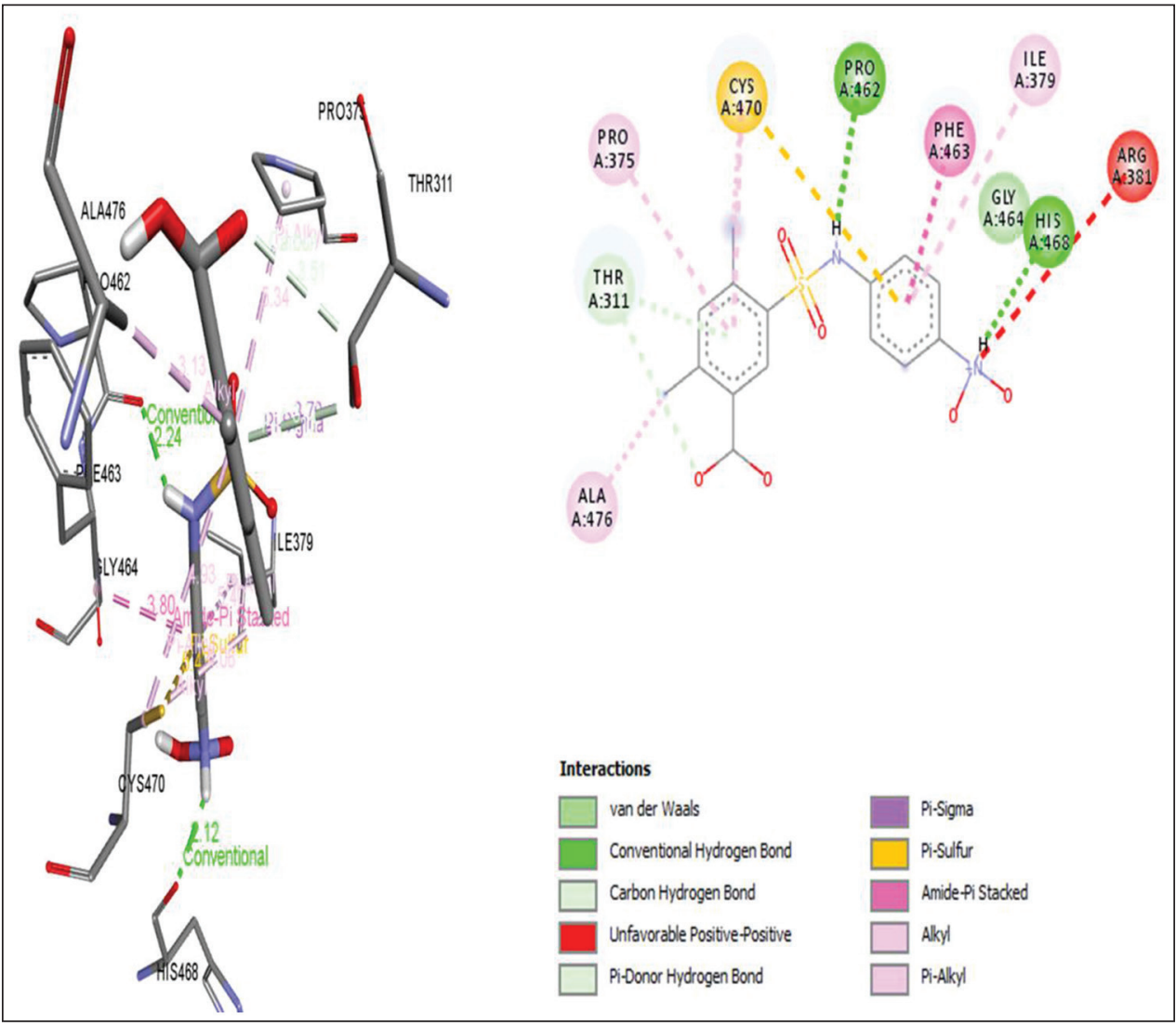

Fig. 2: Binding interactions of compound 1 with active site of 5 fsa

Ala:476, Cys:470 while 2,4-dichloro substituted phenyl ring engaged with Cys:470, Thr:311 amino acid residues by forming pi-alkyl and pisigma interactions, respectively. Gly:464 amino acid residue formed Van der wall interaction with this compound.
In a comparison of a compound containing $\mathrm{NO}_{2}$ substitution, compounds 1,3 , and 14 , amide-pi-stacked interaction was only found in compound 1 , substituted with $\mathrm{p}-\mathrm{NO}_{2}$ group. Compound $2(\mathrm{p}-\mathrm{Cl})$ formed a similar type of interactions as compared to compound $12(\mathrm{p}-\mathrm{F})$ with two more 
interactions such as alkyl and pi-alkyl interaction with Ile:379 and Phe:105 amino acid residues, respectively, that resulted in an increase in antifungal potential. Compounds with aliphatic substitutions such as 15,16 , and 17 , amide-pi-stacked, and pi-pi stacked interactions were not observed which might have contributed for the decrease in antifungal potential.

\section{CONCLUSION}

The set of 18 test compounds was subjected to antimicrobial evaluation and results depicted that compounds with $\mathrm{NO}_{2}$ substitution were most active and para-substituted nitro group effectively increase the antimicrobial potential as compared to substitution at ortho and meta positions. The compounds with chloro substitution were found to be better in activity in comparison to other halogen substituents. The QSAR studies depicted the involvement of lipophilic parameter MR and Balaban index $(\mathrm{J})$ as governors of antimicrobial potential in case of antibacterial and antifungal activity, respectively. Docking studies correlated the activity with structural features by depicting various interaction modes through which the test compounds were interacting with microbial targets such as hydrophobic, hydrogen bonding, and Van der Wall interactions.

\section{ACKNOWLEDGMENTS}

The author (VS) gratefully acknowledge the financial support as a minor project for purchase of chemicals and Junior Research Fellow award to Ms. Samridhi Thakral by Dr. A. P. J. Abdul Kalam Central Instrumentation laboratory, G. J. U. S. and T., Hisar under DST-PURSE Programme. The authors are thankful to Chairman, Department of Pharmaceutical Sciences, G. J. U. S. and T., Hisar for providing necessary facilities to carry out this research work.

\section{AUTHORS' CONTRIBUTIONS}

The authors ST and VS have carried out the in vitro evaluation and computational studies.

\section{CONFLICTS OF INTEREST}

The authors declare that they have no conflicts of interest.

\section{REFERENCES}

1. Lal K, Yadav P, Kumar A, Kumar A, Paul AK. Design, synthesis, characterization, antimicrobial evaluation and molecular modeling studies of some dehydroacetic acid-chalcone-1,2,3-triazole hybrids. Bioorg Chem 2018;77:236-44

2. Thangaraj M, Gengan RM, Ranjan B, Muthusamy R. Synthesis, molecular docking, antimicrobial, antioxidant and toxicity assessment of quinoline peptides. J Photochem Photobiol B 2018;178:287-5.

3. Noreen M, Rasool N, Gull Y, Zahoor AF, Yaqoob A, Kousar S, et al. A facile synthesis of new 5-aryl-thiophenes bearing sulfonamide moiety via Pd (0)-catalyzed suzuki-miyaura cross coupling reactions and 5-bromothiophene-2-acetamide: As potent urease inhibitor, antibacterial agent and hemolytically active compounds. J Saudi Chem Soc 2017;21:S403-14.

4. Swain SS, Paidesetty SK, Padhy RN. Antibacterial activity, computational analysis and host toxicity study of thymol-sulfonamide conjugates. Biomed Pharmacother 2017;88:181-93.

5. Pawar CD, Sarkate AP, Karnik KS, Shinde DB. Synthesis and evaluation of [N-(substituted phenyl)-2-(3-substituted) sulfamoyl) phenyl)] acetamide derivatives as anticancer agents. Egypt J Basic Appl Sci 2017;4:310-4

6. Pomarnacka E, Kozlarska-Kedra I. Synthesis of 1-(6-chloro-1,1-dioxo1,4,2-benzodithiazin-3-yl)semi-carbazides and their transformation into 4-chloro-2-mercapto-N-(4,5-dihydro-5-oxo-4-phenyl-1H-1,2,4triazol-3-yl)benzenesulfonamides as potential anticancer and anti-HIV agents. Farmaco 2003;58:423-9.

7. Ghorab MM, Ragab FA, Hamed MM. Design, synthesis and anticancer evaluation of novel tetrahydroquinoline derivatives containing sulfonamide moiety. Eur J Med Chem 2009;44:4211-7.

8. Zayed MF, Ahmed HE, Ihmaid S, Omar AS, Abdelrahim AS. Synthesis and screening of some new fluorinated quinazolinone-sulphonamide hybrids as anticancer agents. J Taibah Univ Med Sci 2015;10:333-9.

9. Ou L, Han S, Ding W, Jia P, Yang B, Medina-Franco JL, et al. Parallel synthesis of novel antitumor agents: 1,2,3-triazoles bearing biologically active sulfonamide moiety and their 3D-QSAR. Mol Divers 2011;15:927-46.

10. Soyer Z, Uysal S, Parlar S, Tarikogullari Dogan AH, Alptuzun V. Synthesis and molecular docking studies of some 4-phthalimidobenzenesulfonamide derivatives as acetylcholinesterase and butyrylcholinesterase inhibitors. J Enzyme Inhib Med Chem 2017;32:13-9.

11. Riaz S, Khan IU, Bajda M, Ashraf M, Qurat-Ul-Ain, Shaukat A, et al. Pyridine sulfonamide as a small key organic molecule for the potential treatment of Type-II diabetes mellitus and Alzheimer's disease: In vitro studies against yeast $\alpha$-glucosidase, acetylcholinesterase and butyrylcholinesterase. Bioorg Chem 2015;63:64-71.

12. Ranjith PK, Pakkath R, Haridas KR, Kumari SN. Synthesis and characterization of new N-(4-(4-chloro-1H-imidazol-1-yl)3-methoxyphenyl)amide/sulfonamide derivatives as possible antimicrobial and antitubercular agents. Eur J Med Chem 2014;71:354-65.

13. Gadad AK, Noolvi MN, Karpoormath RV. Synthesis and anti-tubercular activity of a series of 2-sulfonamido/trifluoromethyl-6-substituted imidazo[2,1-b]-1,3,4-thiadiazole derivatives. Bioorg Med Chem 2004; $12: 5651-9$

14. Singh K, Sharma PK. Synthesis, characterization and antimicrobial study of some benzenesulfonamide based bipyrazoles. Int J Pharm Pharm Sci 2014;6:345-51.

15. El-Gohary NS, Shaaban MI. Synthesis, antimicrobial, antiquorumsensing, antitumor and cytotoxic activities of new series of fused [1, 3, 4] thiadiazoles. Eur J Med Chem 2013;63:185-95.

16. Abdel-Rahman SA, El-Gohary NS, El-Bendary ER, El-Ashry SM, Shaaban MI. Synthesis, antimicrobial, antiquorum-sensing, antitumor and cytotoxic activities of new series of cyclopenta(hepta)[b] thiophene and fused cyclohepta[b]thiophene analogs. Eur J Med Chem 2017;140:200-11.

17. Kamal A, Swapna P, Shetti RV, Shaik AB, Narasimha Rao MP, Gupta S, et al. Synthesis, biological evaluation of new oxazolidinosulfonamides as potential antimicrobial agents. Eur J Med Chem 2013;62:661-9.

18. Kharbanda C, Alam MS, Hamid H, Javed K, Bano S, Dhulap A, et al. Synthesis and evaluation of pyrazolines bearing benzothiazole as antiinflammatory agents. Bioorg Med Chem 2014;22:5804-12.

19. Kilıcaslan S, Arslan M, Ruya Z, Bilen Ç, Ergün A, Gençer N, et al. Synthesis and evaluation of sulfonamide-bearing thiazole as carbonic anhydrase isoforms hCA I and hCA II. J Enzyme Inhib Med Chem 2016;31:1300-5.

20. Mishra CB, Kumari S, Angeli A, Monti SM, Buonanno M, Prakash A, et al. Design, synthesis and biological evaluation of N-(5-methylisoxazol-3-yl/1,3,4-thiadiazol-2-yl)-4-(3-substitutedphenylureido) benzenesulfonamides as human carbonic anhydrase isoenzymes I, II, VII and XII inhibitors. J Enzyme Inhib Med Chem 2016;31:174-9.

21. Gul HI, Kucukoglu K, Yamali C, Bilginer S, Yuca H, Ozturk I, et al. Synthesis of 4-(2-substituted hydrazinyl)benzenesulfonamides and their carbonic anhydrase inhibitory effects. J Enzyme Inhib Med Chem 2016;31:568-73.

22. Panday V. Modeling of carbonic anhydrase (II) inhibitory activities of sulphonilamide schiff bases by artificial neural network trained with different numerical techniques. Int $\mathrm{J}$ Pharm Pharm Sci 2018;10:202-7.

23. Siddiqui N, Pandeya SN, Khan SA, Stables J, Rana A, Alam M, et al. Synthesis and anticonvulsant activity of sulfonamide derivativeshydrophobic domain. Bioorg Med Chem Lett 2007;17:255-9.

24. Patel TS, Vanparia SF, Patel UH, Dixit RB, Chudasama CJ, Patel BD, et al. Novel 2,3-disubstituted quinazoline-4(3H)-one molecules derived from amino acid linked sulphonamide as a potent malarial antifolates for DHFR inhibition. Eur J Med Chem 2017;129:251-65.

25. Fisher GM, Bua S, Del Prete S, Arnold MS, Capasso C, Supuran CT, et al. Investigating the antiplasmodial activity of primary sulfonamide compounds identified in open source malaria data. Int J Parasitol Drugs Drug Resist 2017;7:61-70.

26. Ugwu DI, Okoro UC, Ukoha PO, Okafor S, Ibezim A, Kumar NM, et al. Synthesis, characterization, molecular docking and in vitro antimalarial properties of new carboxamides bearing sulphonamide. Eur J Med Chem 2017;135:349-69.

27. Thakral S, Singh V. 2,4-dichloro-5-[(N-aryl/alkyl)sulfamoyl]benzoic acid derivatives: In vitro antidiabetic activity, molecular modeling and in silico ADMET screening. Med Chem 2019;15:186-95. 
28. Spooner DF, Sykes G. Laboratory assessment of antibacterial activity. In: Methods in Microbiology. Vol. 7. Ch. 4. London: Academic Press; 1972. p. 211-76.

29. Judge V, Narasimhan B, Ahuja M. Topological models for the prediction of antimycobacterial activity of 4-(5-substituted-1, 3, 4-oxadiazol-2-yl) pyridines. Med Chem Res 2012;21:1363-75.

30. Judge V, Narasimhan B, Ahuja M, Sriram D, Yogeeswari P, De Clercq E, et al. Synthesis, antimycobacterial, antiviral, antimicrobial activities, and QSAR studies of isonicotinic acid-1-(substituted phenyl)-ethylidene/ cycloheptylidene hydrazides. Med Chem Res 2012;21:1935-52.

31. Trott O, Olson AJ. AutoDock vina: Improving the speed and accuracy of docking with a new scoring function, efficient optimization, and multithreading. J Comput Chem 2010;31:455-61.

32. Ghorab MM, Soliman AM, Alsaid MS, Askar AA. Synthesis, antimicrobial activity and docking study of some novel 4-(4, 4-dimethyl-2, 6-dioxocyclohexylidene) methylamino derivatives carrying biologically active sulfonamide moiety. Arab J Chem 2017. DOI: $10.1016 /$ j.arabjc.2017.05.022

33. Zhao S, Wei P, Wu M, Zhang X, Zhao L, Jiang X, et al. Design, synthesis and evaluation of benzoheterocycle analogues as potent antifungal agents targeting CYP51. Bioorg Med Chem 2018;26:3242-53.

34. Szafrański K, Sławiński J, Kędzia A, Kwapisz E. Syntheses of novel 4-substituted N-(5-amino-1H-1,2,4-triazol-3-yl)pyridine-3-sulfonamide derivatives with potential antifungal activity. Molecules 2017;22:E1926. 